\title{
Phytoprotection
}

\section{Comparaison de deux méthodes de mesure de l'efficacité et de la vitesse d'action des défanants sur la pomme de terre}

\section{R. Rioux}

Volume 72, numéro 2, 1991

URI : https://id.erudit.org/iderudit/706003ar

DOI : https://doi.org/10.7202/706003ar

Aller au sommaire du numéro

Éditeur(s)

Société de protection des plantes du Québec (SPPQ)l

ISSN

0031-9511 (imprimé)

1710-1603 (numérique)

Découvrir la revue

Citer cet article

Rioux, R. (1991). Comparaison de deux méthodes de mesure de l'efficacité et de la vitesse d'action des défanants sur la pomme de terre. Phytoprotection, 72(2), 53-60. https://doi.org/10.7202/706003ar
Résumé de l'article

On a comparé les résultats des mesures visuelles et métriques pour l'évaluation de l'efficacité et de la vitesse d'action des défanants de la pomme de terre (Solarium tuberosum) dans trois études conduites en champs à La Pocatière (Québec). Les résultats des deux mesures ont concordé dans 82,5\% des cas pour la question d'évaluer si un effet était statistiquement significatif ou pas au seuil de $5 \%$. La relation entre les deux mesures a été du type linéaire. Pour estimer la vitesse d'action du défanage, un polynôme a été ajusté à la réponse du défanage en fonction du temps et une analyse multivariée a été effectuée sur les coefficients des composantes polynomiales obtenues. Les résultats des deux types de mesures ont concordé dans $100 \%$ des cas. Il n'est donc pas nécessaire d'utiliser des mesures métriques pour exprimer l'efficacité et la vitesse d'action d'un défanant; les mesures visuelles sont tout aussi efficaces. 


\title{
Comparaison de deux méthodes de mesure de l'efficacité et de la vitesse d'action des défanants sur la pomme de terre
}

\author{
Romain Rioux \\ Ferme expérimentale, Agriculture Canada, La Pocatière (Québec), \\ Canada G0R IZO. Contribution No 336
}

(Reçu 1991-02-27; accepté 1991-08-26)

\begin{abstract}
On a comparé les résultats des mesures visuelles et métriques pour l'évaluation de l'efficacité et de la vitesse d'action des défanants de la pomme de terre (Solanum tuberosum) dans trois études conduites en champs à La Pocatière (Québec). Les résultats des deux mesures ont concordé dans 82,5\% des cas pour la question d'évaluer si un effet était statistiquement significatif ou pas au seuil de $5 \%$. La relation entre les deux mesures a été du type linéaire. Pour estimer la vitesse d'action du défanage, un polynôme a été ajusté à la réponse du défanage en fonction du temps et une analyse multivariée a été effectuée sur les coefficients des composantes polynomiales obtenues. Les résultats des deux types de mesures ont concordé dans $100 \%$ des cas. Il n'est donc pas nécessaire d'utiliser des mesures métriques pour exprimer l'efficacité et la vitesse d'action d'un défanant; les mesures visuelles sont tout aussi efficaces.
\end{abstract}

RIOUX, R. 1991. Comparaison de deux méthodes de mesure de l'efficacité et de la vitesse d'action des défanants sur la pomme de terre. PHYTOPROTECTION 72: 53-60.

Visual measurements were compared to metric measures to assess the performance and the rate of potato (Solanum tuberosum) vines desiccation in three field trials conducted at La Pocatière, Québec. The results of the two measures were in agreement in $82.5 \%$ of the cases for the question of testing if an effect was statistically significant or not at the $5 \%$ level. There was a linear relationship between the two methods of measurement. To estimate desiccation rate, a polynomial function was fitted to desiccation response over time and a multivariate analysis of variance was performed on the coefficients obtained. The results of the two types of measurements were in agreement in $100 \%$ of the cases. Thus, it is not necessary to use metric measurements to assess the performance and speed of potato vine desiccants; visual assessments can do the job as well.

\section{Introduction}

La mesure du taux de dessiccation des fanes de pommes de terre (Solanum tuberosum L.) après l'application des défanants est une phase essentielle de la description de leur efficacité. La façon habituelle d'exprimer la vitesse de desséchement est basée sur l'utilisation d'équations de régression (Haderlie et al. 1989; Rioux et Frève 1988). On utilise alors seulement la portion linéaire de la courbe, soit entre le $1^{\text {er }}$ et le $15^{\mathrm{e}}$ jour après le traitement (Haderlie et al. 1989; Rioux et Frève 1988). La vitesse de défanage pourrait être définie plus adéquatement par une courbe décrivant la réduction de la surface foliaire avec le temps pour chaque traitement. On doit cependant disposer de lectures à diverses dates pour décrire cette courbe.

L'efficacité d'un défanant peut aussi être définie selon un niveau de destruction du feuillage ou de la biomasse résiduelle. $\mathrm{Ce}$ - pendant, la période d'évaluation doit être fixée en fonction du mode d'action des produits (Clay 1980). Pour éviter ce problème, l'efficacité des produits est habituellement établie à diverses périodes après le traitement: jusqu'à 14 jours (Bédin 1987; Haderlie et al. 1989; Murphy 1968; Preston 1987) ou 21 jours (Ivany et al. 1986; Stranger 1985). On est donc amené à faire plusieurs observations de la même parcelle.

Les mesures non-destructives étant privilégiées, l'utilisation d'une échelle linéaire de 0 à 100 est fréquente (Ivany et al. 1986). Les résultats sont aussi exprimés en pourcentage de feuilles et de tiges détruites (Rioux et Frève 1988). Avec les herbicides, les mesures visuelles donnent en général le même niveau de réponse que les mesures métriques (Moshier 1987). Cependant, même si les techniques habituelles de l'analyse de variance peuvent être utilisées avec les mesures visuelles (Raymer et al. 1986), et même si la prise de données des mesures métriques est fastidieuse, Clay (1980) recommande d'utiliser les mesures métriques. 
La présente étude vise à comparer deux types de mesures quant à leur capacité à exprimer adéquatement l'efficacité et la vitesse d'action des dessiccants.

\section{Matériel et méthodes}

Les modes d'expression du degré de défanage ont été comparés à partir des données de deux expériences menées en 1982 et 1983 et d'une expérience conduite en 1989. Le mode d'expression de la vitesse de défanage a été examiné à partir des résultats d'une expérience conduite en 1989. Les trois études ont été réalisées à la Ferme expérimentale d'Agriculture Canada à La Pocatière (Québec).

Les deux expériences de 1982 et 1983 ontété réalisées dans le cadre d'une étude portant sur les facteurs affectant l'efficacité de deux produits défanants de la pomme de terre (Rioux et Frève 1988). Dans la première expérience (essai de buses), les traitements étaient définis par deux facteurs à deux niveaux chacun: deux défanants et deux types de buse. On a comparé une buse à fente (Tee Jet 8003) équipant un pulvérisateur portatif et une buse rotative sur un applicateur à gouttelettes contrôlées. Les défanants étaient le glufosinate [(ammonium[3amino-3-carboxy-propyl])methylphosphinate], utilisé en solution concentrée de $200 \mathrm{~g}$ m.a./L (Hoe 39866, Hoechst) et le diquat (dication éthylène-1,1' dipyridylium-2,2'), une solution commerciale de Reglone (200 g m.a./L). Dans la seconde expérience (essai de produit), les deux mêmes défanants ont été appliqués à deux dates différentes. Deux doses d'application ont été utilisées avec le glufosinate $(500$ et $750 \mathrm{~g}$ m.a./ha). Le diquat a été employé à la dose unique de $800 \mathrm{~g} \mathrm{~m}$.a./ha avec ou sans huile minérale (Korn oil SCC581).

Les parcelles de ces deux essais ont été implantées selon un plan en blocs aléatoires complets avec quatre répétitions. Chaque parcelle comprenait trois rangs. Les mesures ont été prises sur le rang central. Un témoin nontraité était inclus dans chacune des expériences. L'effet des herbicides sur la défoliation et la dessiccation des tiges a été mesuré 15 jours après les traitements. Les autres procédures suivies ont été décrites dans Rioux et Frève (1988)

L'efficacité des traitements a été déterminée par une mesure visuelle et par une mesure métrique. Pour obtenir la mesure visuelle, le pourcentage de feuilles et de tiges desséchées a été évalué par rapport aux plantes du témoin non-traité. Le même observateur a fait les mesures pour chaque expérience et à chaque année. Les mesures métriques ont été obtenues en déterminant la longueur non-défanée (verte) des tiges et des feuilles (Benoit et al. 1983). La somme des longueurs de chaque feuille a été utilisée pour estimer la surface foliaire (Epstein et Robinson 1965). Les mesures métriques exigeant beaucoup de temps, il a fallu utiliser seulement deux plantes par parcelle, lesquelles ont été sélectionnées au hasard.

L'expérience de 1989 (essai de maturité) a été réalisée dans le cadre d'une étude sur l'influence des stades de maturité sur l'efficacité du glufosinate. Cette expérience a été réalisée sur un loam graveleux de la série Saint-.André. Des semences de pommes de terre de classe fondation du cultivar Kennebec ont été utilisées. La semence a été plantée à intervalles de 19,5 cm sur des rangs espacés de $90 \mathrm{~cm}$. Chaque parcelle était constituée de trois rangs d'une longueur de $4 \mathrm{~m}$. Chaque année, $120 \mathrm{~kg} /$ ha de $\mathrm{P}_{2} \mathrm{O}_{5}$ et de $\mathrm{K}_{2} \mathrm{O}$ ont été appliqués au semis. Les pratiques culturales étaient faites selon les recommandations du Conseil des productions végétales du Québec (Anonyme 1982, 1983).

Six stades de maturité ont été obtenus en variant les dates de semis et les doses d'azote. L'azote aété appliqué aux doses de 80 et $160 \mathrm{~kg} /$ ha et la plantation a été faite les 12,18 et 26 mai 1989. Pour chaque stade, le glufosinate a été appliqué le 21 juillet, soit 89,97 et 101 jours après la plantation, aux doses de $0,100,200$, 300,400 et $500 \mathrm{~g} \mathrm{~m}$.a./ha. Le dispositif expérimental correspondait à un plan factoriel (trois facteurs) en blocs aléatoires complets avec trois répétitions.

Les mesures de défoliation et de dessiccation des tiges ont été prises sur le rang central à $0,7,14$ et 21 jours après le traitement. Les mesures visuelles ont été faites de la même façon qu'en 1982-1983. Pour les mesures métriques, nous avons utilisé la longueur de la partie verte des tiges comme en 1982-1983. Pour les feuilles, la surface foliaire (verte) a été déterminée au moyen d'un planimètre Li-Cor (modèle LI-3100).

Les données ont été soumises à l'analyse de variance pour déterminer quels effets étaient significatifs au seuil de 5\%. Les résultats obtenus avec les deux types de mesures ont ensuite 
été comparés entre eux afin de déterminer le degré de concordance entre les mesures visuelles et les mesures métriques. Pour stabiliser la variance, les mesures de longueur et de surface foliaire ont fait l'objet d'une transformation arcsinus racine carrée.

En vue de caractériser la relation entre les mesures visuelles et métriques, une régression linéaire a été utilisée. Pour déterminer la régression libre de l'effet des traitements et des répétitions (Cornell et Berger 1987), on a utilisé l'analyse de covariance. En 1982, les analyses de covariance ont montré que la pente de la droite de régression entre les mesures visuelles et métriques n'était pas homogène lorsque le témoin non-traité était inclus. Pour des raisons d'uniformité, toutes les analyses statistiques ont été faites en excluant le témoin.

Pour comparer la vitesse de défanage entre les mesures visuelles et métriques de 1989, on a analysé le degré (\%) de défanage et la surface foliaire résiduelle, en fonction du temps, en utilisant la méthode de régression polynômiale d'Allen et al. (1983). Le procédé consiste, pour chaque unité expérimentale, à ajuster un polynôme à la réponse du défanage par rapport au temps et à effectuer une analyse multivariée sur les coefficients des composantes polynômiales obtenues. Les polynômes de degré J-1 (J étant le nombre de lectures prises après l'application du défanant) ont été ajustés aux données de défanage provenant de chaque parcelle du dispositif expérimental.

Les polynômes ont été examinés et le degré a été choisi de sorte que toutes les réponses soient décrites adéquatement. Par exemple, si quelques parcelles étaient mieux représentées par un polynôme du deuxième degré alors que toutes les autres pouvaient être adéquatement décrites par un polynôme du premier degré, on a choisi le second degré pour analyser l'ensemble des données. Une fois cette sélection faite, les coefficients de régression obtenus ont été soumis à une analyse de variance multivariée en vue de vérifier les hypothèses. Les seuils de probabilité ont été déterminés selon le critère de Wilks.

\section{Résultats et discussion}

Dans les essais de 1982 et 1983, on a observé $82,5 \%$ de concordance entre les résultats d'analyse de variance effectués avec les mesures visuelles et les mesures métriques (tableau 1). Dans la plupart des cas, l'interprétation de l'effet des facteurs a donc été la même pour les deux types de mesures. Les coefficients de

Tableau 1. Concordance des résultats d'analyses de variance effectuées avec les mesures visuelles et métriques dans trois expériences de défanage de la pomme de terre

\begin{tabular}{lccc}
\hline Expérience & $\begin{array}{c}\text { Nombre de tests F } \\
\text { effectués avec chaque } \\
\text { type de mesure }\end{array}$ & $\begin{array}{c}\text { Nombre de tests F pour lesquels } \\
\text { les mesures visuelles et } \\
\text { métriques concordent }\end{array}$ & $\begin{array}{c}\text { Concordance } \\
(\%)\end{array}$ \\
\hline Type de buses & 6 & 6 & 100,0 \\
1982 & 6 & 5 & 83,3 \\
1983 & 12 & 11 & 91,6 \\
$1982-1983$ & & & 71,4 \\
Produit & 14 & 10 & 85,7 \\
1982 & 14 & 12 & 78,5 \\
1983 & 28 & 22 & 82,5 \\
1982-1983 & 40 & 33 & 85,7 \\
Total 1982-1983 & & & 85,7 \\
Maturité (1989) & 14 & 12 & 92,8 \\
Évaluation 7 JAT & 14 & 12 & 88,1 \\
Évaluation 14 JAT & 14 & 13 & 85,4 \\
Évaluation 21 JAT & 42 & 37 & \\
Total 1989 & 82 & 70 & \\
Grand total & & &
\end{tabular}

$\S$ JAT: Jours après le traitement. 
détermination ont été légèrement inférieurs $(0,6856 \pm 0,1440$ vs $0,7153 \pm 0,1954)$ et les coefficients de variation nettement plus élevés $(39,15 \pm 21,82$ vs $21,68 \pm 11,24)$ avec les mesures métriques. L'échantillonnage destructif a introduit une source additionnelle d'erreur par rapport aux mesures visuelles puisque seulement deux plantes sont évaluées. Holstun et McWhorter (1961) ont obtenu des résultats semblables lors d'essais de désherbants chimiques.

Dans l'essai de comparaison de types de buse, les analyses de variance ont suggéré les mêmes interprétations pour les mesures visuelles et pour les mesures métriques dans $91,6 \%$ des cas. Dans l'essai de comparaison de défanants, les analyses de variance ont suggéré les mêmes interprétations pour les deux types de mesures dans $78 \%$ des cas (tableau 1). Dans cet essai, il y a plus de différence entre les types de mesure en 1983 qu'en 1982. Le manque de concordance peut provenir en partie de la façon de mesurer. Lorsque le temps entre le traitement et la lecture est court, il est difficile de mesurer de petites différences dans la longueur des parties vertes des feuilles ou des tiges. Cependant une différence de couleur ou de turgidité peut facilement être évaluée et être incluse dans la lecture visuelle. Toutefois, lors del'utilisation de ce dernier type de mesure, l'estimateur doit porter un jugement, qui sera nécessairement empreint d'une certaine subjectivité, et qui peut devenir une source de biais (Holstun et McWhorter 1961).

Dans l'essai de maturité, d'après les analyses de variance, les mêmes interprétations pouvaient être faites pour les mesures visuelles et pour les mesures métriques dans $88,1 \%$ des cas (tableau 1). Les mesures métriques ont généralement des coefficients de variation plusélevés $(39,15 \pm 21,82$ vs $21,65 \pm 11,24)$ que les mesures visuelles. Cette particularité, tel que souligné plus haut, pourrait être due à l'échantillonnage réduit à deux plants. La plupart des cas de discordance impliquait l'azote. À 7 jours après le traitement (JAT), les mesures métriques pour les tiges indiquaient une longueur totale de tiges vertes de $756 \mathrm{~cm}$ à $160 \mathrm{~kg} / \mathrm{ha}$ d'azote comparativement à $636 \mathrm{~cm}$ à $80 \mathrm{~kg} / \mathrm{ha}$. Les mesures visuelles indiquaient dans les deux cas moins de $1 \%$ de défanage. Le résultat du test $\mathrm{F}$ de la mesure métrique indique que l'azote a davantage influencé la hauteur des tiges que l'effet des défanants (données non présentées). La plantation tardive et l'application d'azote ont résulté en des plants conservant leur surface foliaire plus longtemps. Suite aux traitements de défanage, il y a eu des différences significatives entre la réaction des fanes immatures et matures même si leur apparence est, dans les deux cas, verte et saine (Haderlie et al. 1989). Les fanes immatures ont besoin d'une plus longue période pour atteindre une dessiccation complète et ceci est particulièrement vrai pour les tiges (Rioux 1983).

Dans l'essai sur les types de buses, les mesures métriques ont montré une forte relation linéaire avec les mesures visuelles (tableau 2). La relation a été significative sauf pour les feuilles en 1982. Le test d'homogénéité des coefficients de régression a indiqué qu'il n'y avait pas de différence dans la pente entre les traitements. Dans l'essai de produits, les mesures métriques ont aussi été reliées linéairement aux mesures visuelles à chacune des années d'étude (tableau 2). Les pentes des droites de régression des mesures visuelles sur les mesures métriques étaient homogènes. Dans l'essai de 1989 sur la maturité, la relation entre la mesure métrique et la mesure visuelle a également pu être exprimée par une relation linéaire pour chaque date d'évaluation (tableau 2). À 7 jours après le traitement (JAT), la pente n'était pas significative pour les tiges et les coefficients de détermination étaient peu élevés pour les feuilles et pour les tiges. Les coefficients de détermination ont augmenté lorsque l'évaluation s'est faite plus tard en saison, soit à 14 et 21 JAT. Les mesures visuelles ont donc été un meilleur indicateur de la réduction de la surface foliaire lorsque les dommages ont été évalués 21 JAT. Clay (1980) avait déjà souligné l'importance du moment de la prise de données avec les herbicides.

Dans tous les cas, la relation entre les mesures visuelles et les mesures métriques a été de type linéaire et le plus souvent les deux mesures mènent aux mêmes conclusions. Lorsqu'il y avait des différences entre les mesures, un facteur de maturité était généralement en cause et ces différences ont surtout été importantes les premiers jours après les traitements. La mesure visuelle pourrait être influencée par des facteurs de couleur et de turgescence, facteurs dont une mesure de surface foliaire ne tiendrait pas compte. 
Tableau 2. Relation entre les mesures visuelles $(\mathrm{y}=\arcsin \sqrt{\%+0,1})$ et les mesures métriques $(\mathrm{x}=\sqrt{\mathrm{cm}+0,1})^{\S}$ dans trois expériences de défanage de la pomme de terre

\begin{tabular}{|c|c|c|c|c|c|c|}
\hline \multicolumn{2}{|c|}{ Expérience } & Équation de régression & $P>\mathrm{F}^{\dagger}$ & $P>|\mathrm{T}|^{\stackrel{⿱}{*}}$ & $\begin{array}{c}\text { Coefficient } \\
\text { de détermination }\end{array}$ & $\begin{array}{c}\text { Coefficient } \\
\text { de variation }(\%)\end{array}$ \\
\hline \multicolumn{7}{|c|}{ Type de buse $(n=16)$} \\
\hline Feuilles & 1982 & $y=1,3720-0,0099 x$ & 0,0292 & 0,5294 & 0,7314 & 12,38 \\
\hline Feuilles & 1983 & $y=1,5015-0,0305 x$ & 0,0001 & 0,0012 & 0,9678 & 9,69 \\
\hline Tiges & 1982 & $y=1,3931-0,0401 x$ & 0,0002 & 0,0064 & 0,9444 & 11,12 \\
\hline Tiges & 1983 & $y=1,4171-0,0375 x$ & 0,0001 & 0,0038 & 0,9793 & 19,75 \\
\hline \multicolumn{7}{|c|}{ Produit ( $n=24)$} \\
\hline Feuilles & 1982 & $y=1,5387-0,0180 x$ & 0,0422 & 0,0013 & 0,9026 & 4,28 \\
\hline Feuilles & 1983 & $y=0,9940-0,0105 x$ & 0,0318 & 0,0658 & 0,9594 & 10,49 \\
\hline Tiges & 1982 & $y=1,5571-0,0342 x$ & 0,0006 & 0,0002 & 0,9463 & 10,38 \\
\hline Tiges & 1983 & $\mathrm{y}=1,0319-0,0411 \mathrm{x}$ & 0,0043 & 0,0060 & 0,9813 & 18,23 \\
\hline \multicolumn{7}{|c|}{ Maturité $(n=90)$} \\
\hline Feuilles & $7 \mathrm{JAT}^{\mathbb{1}}$ & $y=0,7556-0,0017 x$ & 0,0001 & 0,0001 & 0,3433 & 16,01 \\
\hline Feuilles & 14 JAT & $y=1,4075-0,0076 x$ & 0,0001 & 0,0001 & 0,6818 & 16,40 \\
\hline Feuilles & $21 \mathrm{JAT}$ & $y=1,5092-0,0057 x$ & 0,0001 & 0,0001 & 0,6891 & 19,04 \\
\hline Tiges & 7 JAT & $y=0,0998-0,0011 x$ & 0,1223 & 0,2645 & 0,1262 & 111,37 \\
\hline Tiges & 14 JAT & $y=1,1568-0,0222 x$ & 0,0001 & 0,0001 & 0,4045 & 41,21 \\
\hline Tiges & $21 \mathrm{JAT}$ & $y=1,3264-0,0259 x$ & 0,0001 & 0,0001 & 0,7269 & 21,52 \\
\hline
\end{tabular}

$\S$ Pour les feuilles, dans l'essai de maturité, $\mathrm{x}=\sqrt{\mathrm{cm}^{2}+0,1}$.

$\dagger$ Degré de signification de l'équation (test F).

$\ddagger$ Degré de signification de la pente (test $\mathrm{T}$ ).

I JAT: jours après le traitement. 
Tableau 3. Résultats des analyses multivariées $\$$ effectuées avec les mesures visuelles et les mesures métriques dans l'essai de 1989, visant à déterminer l'influence des doses de glufosinate, des doses d'azote, et de: la précocité du traitement sur le défanage de la pomme de terre

\begin{tabular}{|c|c|c|}
\hline \multirow[b]{2}{*}{ Facteur } & \multicolumn{2}{|c|}{ Seuil de probabilité selon le critère de Wilks } \\
\hline & Mesures visuelles & Mesures métriques; \\
\hline \multicolumn{3}{|l|}{ Feuilles } \\
\hline Glufosinate $(\mathrm{G})$ & 0,0009 & 0,0131 \\
\hline Azote $\quad(\mathrm{N})$ & 0,8719 & 0,4678 \\
\hline Précocité (P) & 0,0048 & 0,0001 \\
\hline $\mathrm{G} \times \mathrm{N}$ & 0,3404 & 0,1726 \\
\hline$G \times P$ & 0,6087 & 0,2196 \\
\hline $\mathrm{N} \times \mathrm{P}$ & 0,0249 & 0,0228 \\
\hline $\mathrm{G} \times \mathrm{N} \times \mathrm{P}$ & 0,2952 & 0,0838 \\
\hline \multicolumn{3}{|l|}{ Tiges } \\
\hline Glufosinate $(\mathrm{G})$ & 0,0293 & 0,0181 \\
\hline Azote $\quad(\mathrm{N})$ & 0,8898 & 0,7998 \\
\hline Précocité (P) & 0,0006 & 0,0141 \\
\hline $\mathrm{G} \times \mathrm{N}$ & 0,4164 & 0,7997 \\
\hline $\mathrm{G} \times \mathrm{P}$ & 0,1737 & 0,6276 \\
\hline $\mathrm{N} \times \mathrm{N} \times \mathrm{P}$ & 0,0066 & 0,0360 \\
\hline$G \times N \times P$ & 0,9028 & 0,1788 \\
\hline
\end{tabular}

$\S$ Les analyses ont été effectuées sur les coefficients de régression (réponse du défanage par rapport au temps) qui caractérisent chaque unité expérimentale.

Tableau 4. Équations de régression $\$$ illustrant la relation entre la dessiccation du feuillage de la pomme de terre ${ }^{\dagger}(\mathrm{y})$ et le temps ${ }^{\ddagger}(\mathrm{x})$, telle qu'affectée par les doses de glufosinate, et par la précocité à différentes doses d'azote

\begin{tabular}{lccc}
\hline & & \multicolumn{2}{c}{ Équation de régression } \\
\cline { 2 - 4 } Facteur & \multicolumn{1}{c}{ Mesures visuelles } & Mesures métriques \\
\hline $\begin{array}{l}\text { Glufosinate }(g / h a) \\
100\end{array}$ & $\mathrm{y}=0,0273+0,0775 \mathrm{x}-0,0011 \mathrm{x}^{2}$ & \\
200 & $\mathrm{y}=0,0273+0,0887 \mathrm{x}-0,0015 \mathrm{x}^{2}$ & $\mathrm{y}=103,9-3,32 \mathrm{x}-0,01 \mathrm{x}^{2}$ \\
300 & $\mathrm{y}=0,0273+0,1032 \mathrm{x}-0,0019 \mathrm{x}^{2}$ & $\mathrm{y}=103,9-5,10 \mathrm{x}+0,07 \mathrm{x}^{2}$ \\
400 & & $\mathrm{y}=0,0273+0,1146 \mathrm{x}-0,0024 \mathrm{x}^{2}$ & $\mathrm{y}=103,9-6,22 \mathrm{x}+0,12 \mathrm{x}^{2}$ \\
500 & & $\mathrm{y}=0,0273+0,1033 \mathrm{x}-0,0019 \mathrm{x}^{2}$ & $\mathrm{y}=103,9-6,75 \mathrm{x}+0,13 \mathrm{x}^{2}$ \\
Azote & Précocité & & $\mathrm{y}=103,9-5,82 \mathrm{x}+0,08 \mathrm{x}^{2}$ \\
$(\mathrm{~kg} / \mathrm{ha})$ & $\mathrm{JAP}$ & $\mathrm{y}=0,0298+0,0869 \mathrm{x}-0,0014 \mathrm{x}^{2}$ & \\
80 & 89 & $\mathrm{y}=0,0399+0,0864 \mathrm{x}-0,0014 \mathrm{x}^{2}$ & $\mathrm{y}=103,9-2,72 \mathrm{x}-0,04 \mathrm{x}^{2}$ \\
80 & 97 & $\mathrm{y}=0,0089+0,1254 \mathrm{x}-0,0027 \mathrm{x}^{2}$ & $\mathrm{y}=103,9-4,84 \mathrm{x}-0,06 \mathrm{x}^{2}$ \\
80 & 101 & $\mathrm{y}=0,0329+0,0875 \mathrm{x}-0,0013 \mathrm{x}^{2}$ & $\mathrm{y}=103,9-10,14 \mathrm{x}+0,25 \mathrm{x}^{2}$ \\
160 & 89 & $\mathrm{y}=0,0222+0,0988 \mathrm{x}-0,0018 \mathrm{x}^{2}$ & $\mathrm{y}=103,9-4,33 \mathrm{x}+0,02 \mathrm{x}^{2}$ \\
160 & 97 & $\mathrm{y}=0,0299+0,0997 \mathrm{x}-0,0019 \mathrm{x}^{2}$ & $\mathrm{y}=103,9-6,11 \mathrm{x}+0,13 \mathrm{x}^{2}$ \\
160 & 101 & $\mathrm{y}=103,9-4,53 \mathrm{x}+0,04 \mathrm{x}^{2}$ \\
\hline
\end{tabular}

§Un polynôme du deuxième degré a été ajusté aux données de chaque unité expérimentale et une analyse multivariée a été effectuée sur les coefficients de régression obtenus (tableau 3). Les équations présentées ici ont été obtenues en regroupant l'ensemble des unités expérimentales correspondant à une dose de glufosinate ou à une combinaison: dose d'azote - précocité (soit les facteurs significatifs selon l'analyse multivariée).

$\dagger$ Pour les mesures visuelles, $y=\arcsin \sqrt{\%+0,1}$; pour les mesures métriques, $\mathrm{y}=\sqrt{\mathrm{cm}^{2}+0,1}$

† Jours après le traitement.

I Jours après la plantation. 
Dans l'essai de maturité, les analyses multivariées ont suggéré les mêmes interprétations pour les deux types de mesure: l'effet du glufosinate, de la précocité, et de l'interaction azoteprécocité, se sont avérés significatifs pour les feuilles et pour les tiges (tableau 3 ). Le progrès de la dessiccation des feuilles a pu être décrit adéquatement par un polynôme du deuxième degré pour les deux types de mesures (tableau 4). La vitesse de défanage a augmenté avec la dose de glufosinate jusqu'à $400 \mathrm{~g} / \mathrm{ha}$. À faible dose d'azote, la vitesse de défanage a aussi augmenté lorsque les traitements ont été appliqués plus tardivement. À dose élevée d'azote, l'effet de la précocité du traitement a disparu. Haderlie et al. (1989) avaient déjà montré que des fanes matures sont plus faciles à dessécher que des fanes immatures. Il y a une différence significative dans la réaction aux dessiccants des fanes matures comparativement aux fanes immatures et des fanes traitées à doses élevées d'azote comparativement à des doses faibles (Rioux 1983).

La dessiccation des tiges a été retardée par rapport à celle des feuilles (données non présentées), de telle sorte qu'aucun signe de dessicca- tion n'avait été enregistré 7 jours après le traitement. Le progrès de la dessiccation des tiges a donc été décrit entre 7 et 21 JAT plutôt qu'entre 0 et 21 JAT. La relation entre la dessiccation des tiges de la pomme de terre et le temps s'est donc avérée linéaire (tableau 5), plutôt que quadratique comme dans le cas des feuilles. Toutefois, la vitesse de dessiccation des tiges a été influencée par les mêmes facteurs que la vitesse de dessiccation du feuillage, soit par les doses de glufosinate, et par la précocité à faible dose d'azote.

La concordance entre les deux types de mesures a été suffisante pour que nous puissions affirmer que les mesures visuelles, tout autant que les mesures métriques, peuvent nous permettre d'exprimer adéquatementl'efficacité des dessiccants. L'analyse de la vitesse d'action nous a permis d'atteindre les mêmes conclusions avec les deux types de mesures. Cette étude nous permet donc de conclure qu'on peut utiliser les mesures visuelles pour décrire l'efficacité et la vitesse d'action des défanants, sans affecter la crédibilité des résultats; il n'est donc pas nécessaire de s'astreindre à prendre des mesures métriques.

Tableau 5. Équations de régression $\$$ illustrant la relation entre la dessiccation des tiges de la pomme de terre ${ }^{\dagger}(\mathrm{y})$ et le temps ${ }^{*}(\mathrm{x})$, telle qu'affectée par les doses de glufosinate, et par la précocité à différentes doses d'azote

\begin{tabular}{|c|c|c|c|}
\hline \multicolumn{2}{|l|}{ Facteur } & Mesures visuelles & Mesures métriques \\
\hline \multicolumn{4}{|c|}{ Glufosinate (g/ha) } \\
\hline 100 & & $y=-0,3577+0,0543 x$ & $y=39,5-0,9425 x$ \\
\hline 200 & & $y=-0,3577+0,0576 x$ & $y=39,5-0,9345 x$ \\
\hline 300 & & $y=-0,3577+0,0621 x$ & $y=39,5-0,8917 x$ \\
\hline 400 & & $y=-0,3577+0,0668 x$ & $y=39,5-1,2061 x$ \\
\hline 500 & & $y=-0,3577+0,0703 x$ & $y=39,5-1,3773 x$ \\
\hline $\begin{array}{c}\text { Azote } \\
\text { (kg/ha) }\end{array}$ & $\begin{array}{c}\text { Précocité } \\
\text { JAP }\end{array}$ & & \\
\hline 80 & 89 & $y=-0,3577+0,0525 x$ & $y=40,7-1,0094 x$ \\
\hline 80 & 97 & $y=-0,3577+0,0585 x$ & $y=35,2-0,6470 x$ \\
\hline 80 & 101 & $y=-0,3577+0,0783 x$ & $y=41,4-1,5404 x$ \\
\hline 160 & 89 & $y=-0,3577+0,0609 x$ & $y=40,6-1,0968 x$ \\
\hline 160 & 97 & $y=-0,3577+0,0622 x$ & $y=35,0-0,8107 x$ \\
\hline 160 & 101 & $y=-0,3577+0,0613 x$ & $y=44,2-1,3182 x$ \\
\hline
\end{tabular}

§ Un polynôme du premier degré a été ajusté aux données de chaque unité expérimentale et une analyse multivariée a été effectuée sur les coefficients de régression obtenus (tableau 3). Les équations présentées ici ont été obtenues en regroupant l'ensemble des unités expérimentales correspondant à une dose de glufosinate ou à une combinaison: dose d'azote - précocité (soit les facteurs significatifs selon l'analyse multivariée).

$\dagger$ Pour les mesures visuelles, $\mathrm{y}=\arcsin \sqrt{\%+0,1}$; pour les mesures métriques. $\mathrm{y}=\sqrt{\mathrm{cm}^{2}+0,1}$.

$\doteqdot$ Jours après le traitement.

I Jours après la plantation. 
L'auteur désire remercier $\mathrm{M}^{\mathrm{mc}}$ Hélène Massé pour son aide à la présentation du texte ainsi que $\mathrm{M}^{\text {me }}$ Michèle Bernier-Cardou qui a collaboré à l'analyse statistique des résultats.

Allen, O.B., J.M. Burton et J.O. Holtz. 1983. Analysis of repeated measurements using polynomial regression. J. Anim. Sci. 57: 765-770.

Anonyme. 1982. Pomme de terre-guide de protection. Conseil des productions végétales du Québec. Ministère de l'Agriculture, des Pêcheries et de l'Alimentation du Québec. Agdex 161/605. 31 pp.

Anonyme. 1983. Pomme de terre-guide de culture. Conseil des productions végétales du Québec. Ministère de l'Agriculture, des Pêcheries et de l'Alimentation du Québec. Agdex 161/20. 39 pp.

Bédin, P. 1987. Le défanage des cultures de pommes de terre. La pomme de terre française, no. 441 (juilletaoût), p. 175-177.

Benoit, G.R., C.D. Stanley, W.G. Grant et D.B. Torrez. 1983. Potato top growth as influenced by temperatures. Am. Potato J. 60: 489-501.

Clay, D.V. 1980. Indices and criteria for comparing the tolerance of strawberries to herbicides in dose-response experiments. Weed Res. 20: 91-96.

Cornell, J.A. et R.D. Berger. 1987. Factors that influence the value of the coefficient of determination in simple linear and non linear regression models. Phytopathology 77: 63-70.
Epstein, E. et R.R. Robinson. 1965. A rapid method for determining leaf area of potato plants. Agron. J. 57: 515-516.

Haderlie, L.C., J.L. Halderson, P.W. Leino, P.J. Peterson et R.H. Callihan. 1989. Chemical desiccation of potato vines. Am. Potato J. 66: 53-62.

Holstun, J.T., Jr. et C.G. McWhorter. 1961. Methods of evaluating pre-emergence herbicides for cotton. Weeds 9: 527--537.

Ivany, J.A., R.P. White et J.B. Sanderson. 1986. Effect of applied fertilizer on Kennebec potato top desiccation. Am. Potato J. 63: 545-552.

Moshier, L.G. 1987. Shattercane (Sorghum bicolor) control with alachlor where EPTC failed. Weed Technol. 1: 231-234.

Murphy, H.G. 1968. Potato vine killing. Am. Potato J. 45: 472-478.

Preston, D. 1987. Vine killing. Valley Potato Grower (August). p. 7-8.

Raymer, J.C.W., K.G. Dodds et D.J. Best. 1986. The effect of categorization on a simple analysis of variance model. Biom. J. 28: 159-170.

Rioux, R. 1983. Effets de la fertilisation azotée, de l'éthéphon et de l'huile minérale sur le cléfanage de la pomme de terre de semence avec le diquat. Phytoprotection 64: 27-30.

Rioux, R. et A. Frève. 1988. Évaluation du glufosinate pour le défanage de la pomme de terre. Phytoprotection 69: 121-125.

Stranger, C.E. 1985. Potato vine desiccation study. SDEC Rep. Oregon State Univ. Agric. Exp. Stn. p. 98-100. 